Although it was the first nuclear process studied by Rutherford, alpha decay is still not properly understood. A large number of alpha decay rates have now been measured for heavy nuclei, and these depend basically on two factors, a nuclear structure factor that gives the probability that there is an alpha particle in the nuclear surface 'waiting to get out', and a penetration factor that gives the probability that it will be able to tunnel quantum-mechanically through the barrier and so escape from the nucleus.

Ever since Gamow first applied quantum mechanics to the nucleus, it has been known that the penetration probability depends very critically on the height and width of the potential barrier through which the alpha particle has to tunnel, and hence on the energy of the emitted alpha particle. This is why the alpha decay halflives vary from a very small fraction of a second to many thousands of years, while the corresponding alpha energies vary by less than an order of magnitude.

This simple theory was very successful in accounting for the relative alpha decay rates of a large number of radioactive nuclei, but the absolute values were much less certain, due to the lack of knowledge of the nuclear structure and of the potential barrier. Using the best available nuclear structure information, there was usually a factor of a thousand between the measured and calculated decay rates and it was generally considered that this large factor was still within the uncertainties of the potentials.

This point has now been carefully checked by De Vries at Rochester and Lilley and Franey at Minnesota (Phys. Rev. Lett., 37, 481; 1976). They

\section{Absolute alpha decay rates}

from P. E. Hodgson

studied the interaction of alpha particles with ${ }^{208} \mathrm{~Pb}$ and ${ }^{200} \mathrm{Bi}$ and fitted the experimental data for elastic scattering at $22 \mathrm{MeV}$ and the reaction cross section from 17 to $24 \mathrm{MeV}$ by a series of optical model potentials. The reaction cross sections are known accurately since they are the sum of the $(\alpha, n),(\alpha, 2 n)$ and $(\alpha, \gamma)$ cross sections, all of which can be measured accurately. No other reactions are energetically possible.

They found that this data imposes tight restrictions on the optical potentials, so that an acceptable fit can only be obtained with parameters constrained within narrow limits. The penetrabilities calculated from these potentials vary somewhat, but far less than was previously thought. This makes it possible to calculate accurate absolute values of the quantity called the reduced alpha width $\gamma_{\alpha}^{2}$ that contain the nuclear structure information alone. It is found that these values are still about a thousand times greater than those calculated from the shell model. For example, for ${ }^{210} \mathrm{Po}$, the value of $\gamma^{2}{ }_{\alpha}$ found from the measured alpha decay rate and the calculated penetrability is 0.88 ${ }_{-0.19}^{+4.4}$, whereas the value calculated from the shell model by Harada is only $(0.31$ to 3.3$) \times 10^{-3}$. Results for other nuclei are similar.

This is a serious discrepancy and shows the need for much more sophisticated nuclear structure calculations, including more configuration mixing and the renormalisation required by antisymmetrisation. A thousand times more alpha particles are emitted than would be expected from existing shell model calculations, so it may well be that this is due to substantial clustering of alpha particles on the nuclear surface that cannot yet be described by the shell model. the climatic fluctuations was not appreciated either (indeed Hinton was a monoglacialist, and so assigned faunas either to "the" cold period or to the preceeding warm period), with the result that much ecological and stratigraphical information was overlooked or lost.

Biological aspects of Pleistocene study have been revolutionised first by pollen analysis and more recently by the study of insect, especially beetle, faunas; physical aspects of study have added absolute chronologies (from radiocarbon and other dating techniques) and absolute temperature calibration (from oxygen isotope ratios). In this surge of knowledge, Pleistocene mammals have, at least in Britain, been left far behind; Zeuner's book (The Pleistocene Period, Hutchinson, 1944) contained a chapter of 30 pages on mammals, but only four single-line entries on pollen analysis, whereas West (Pleistocene Geology and Biology, Longman, 1968), has 32 pages on pollen stratigraphy but only six on mammals.

Clearly the time was ripe for restudy of British Pleistocene mammals, not only in their own right but also to integrate them into the newer framework of Quaternary chronology. Some significant signs of such restudy are now available. One is Stuart's review relating all available vertebrate occurrences to the pollen stratigraphy of Britain (Biol. Rev., 49, 225-266; 1974). this involved new collecting from sites where information on pollen was available or obtainable as well as a reexamination of a scattered and often obsolete literature. Another equally valuable review of a different type is Maglio's paper on the evolution of the elephants (Trans. Am. phil. Soc., 63, $1-149 ; 1973)$. Because they are so conspicuous, fossil elephants, or at least their molars, have played an important part in stratigraphy, but their taxonomy has been extremely muddled.

Perhaps the most important such contribution has just been published by Sutcliffe and Kowalski (Pleistocene Rodents of the British Isles, Bull Br. Mus. (nat. Hist.) Geol. 27, 31-147; 1976). This account is important in a number of respects. For a start, the rodents are, and have been throughout the Pleistocene, the most numerous mammals; they are therefore the most abundant in cave deposits, and so potentially the most useful archaeologically or stratigraphically, and they are arguably the most important ecologically. Their potential has however been largely unrealised, partly perhaps be- cause archaeologists have tended to ignore them, but largely because, as a result of the early studies, they were in a taxonomically unsatisfactory state and therefore unreliable stratigraphically. This review should at least resolve these last obstructions. The literature records are thoroughly reviewed, and their stratigraphical assignments reassessed. The taxonomy is also reviewed, so that it is now much clearer just how many distinct species were present in the Pleistocene. The dubious taxa erected by Hinton, among others, are thoroughly scrutinised, and many are relegated to synonymy. Of particular importance is the cautious reassessment of Microtus species, especially $M$. arvalis. This vole has a rather southern distribution in Europe, being absent from Scandinavia and, except for Orkney and Guernsey, from Britain. The occurrence of fossil remains attributed to it, however (often as $M$. corneri) have led to suggestions that it was an early postglacial immigrant, since isolated as a relict in Orkney. Not only is this inherently unlikely on ecological grounds (human introduction being more likely an explanation) but, as this review makes clear, the supposed fossil occurrences are very doubtful indeed. The 